\title{
ANTICUERPOS DE Leptospira interrogans (sensu lato) EN TRABAJADORES DE GRANJAS PORCINAS DEL MEDIO SINÚ, CÓRDOBA (COLOMBIA) ¿ENFERMEDAD PROFESIONAL?
}

\author{
ANTIBODIES OF Leptospira interrogans (sensu lato) IN PIG FARMS WORKERS \\ IN THE MEDIUM SINU, CORDOBA (COLOMBIA). OCCUPATIONAL DISEASE?
}

\author{
CALDERÓN ALFONSO, ${ }^{*}$ M.Sc, RODRÍGUEZ VIRGINIA, ${ }^{2}$ M.Sc, GUZMÁN, CAMILO,${ }^{3}$ M.Sc. \\ 'Universidad de Córdoba, Facultad de Medicina Veterinaria y Zootecnia, \\ Instituto de Investigaciones Biológicas del Trópico (IIBT), Colombia. \\ ${ }^{2}$ Universidad de Córdoba, Programa de Bacteriología, Grupo De Investigaciones Microbiológicas y \\ Biomédicas de Córdoba (GIMBIC), Colombia. \\ ${ }^{3}$ Universidad de Córdoba, Facultad Ciencias de la Salud. Colombia.
}

\section{Key words:}

Human, infection, pigs farms, seroprevalence, titles, workers, zoonoses.

\section{Abstract}

Leptospirosis is a reemerging zoonotic character, worldwide distribution and with different symptoms, high incidence in tropical regions, where the reservoirs are usually wild and domestic animal urine excreted by the microorganism and are the source of contamination for humans and other animals to be a serious public health problem. It is also considered as an occupational disease because of its high risk of exposure. In the human symptoms of infection are varied and in many cases are unrecognized. The overall objective was to know the seroprevalence of antibodies to Leptospira interrogans (sensu lato) and by a transverse epidemiological study in 62 workers of pigs farms in three municipalities of the medium Sinu blood samples and the completion of the MAT were collected, was implemented with 14 serogroups. The prevalence in workers was $75.81 \%$ and serogroups that presented the highest frequencies were: Canicola, Pomona, Icterohaemorrhagiae and Grippothyphosa.

\section{Palabras Clave:}

Humanos, infección, granjas porcinas, seroprevalencia, títulos, trabajadores, zoonosis.

\section{Resumen}

La leptospirosis, es una zoonosis de carácter reemergente, de distribución mundial y con diversa sintomatología, de gran incidencia en las regiones tropicales, donde los reservorios suelen ser animales domésticos y salvajes que excretan por la orina el microorganismo y son la fuente de contaminación para humanos y otros animales, siendo un serio problema de salud pública. También es considerada como enfermedad profesional por su alto riesgo de exposición. En el humano, la sintomatología de la infección es variada y en muchos casos pasa desapercibida. El objetivo general fue conocer la seroprevalencia de anticuerpos de Leptospira interrogans (sensu lato) y por medio de un estudio epidemiológico transversal en 62 trabajadores de granjas porcinas en tres municipios del medio Sinú, se recolectaron muestras de sangre y la realización del MAT se implementó con 14 serogrupos. La seroprevalencia en los trabajadores fue del $75,81 \%$ y los serogrupos que presentaron las mayores frecuencias fueron: Canicola, Pomona, Icterohaemorrhagiae y Grippothyphosa. 
Introducción

La leptospirosis es una de las zoonosis, de amplia distribución en el mundo; es una enfermedad reemergente, de gran incidencia en las regiones tropicales debido a factores ambientales, climáticos y sociales que favorecen la transmisión (GUERRA, 2013). Hoy día se constituye en un serio problema de Salud Pública, en aquellas regiones con un saneamiento básico inadecuado (NÁJERA et al., 2005).

Los reservorios de este patógeno suelen ser animales domésticos y salvajes que tienen la capacidad de ser hospederos primarios o accidentales, dependiendo del serovar y pueden mantener viable y excretar por la orina, siendo la fuente de contaminación para humanos y otros animales (MONAHAN et al., 2009); las ratas constituyen el principal reservorio en ambientes urbanos y fueron los primeros en ser reconocidos como portadores de leptospiras que se han relacionado con infección en humanos (VIJAYACHARY et al., 2008). Son reservorios primarios para los serovares icteohaemorrhagiae y accidentales para Pomona (DAMMERT, 2005). Los caninos son reservorios primarios para los serovares: Canicola y Bataviae. Los gatos son reservorios primarios de los serovares Hardjo-bovis, Pomona y Grippothyphosa y los porcinos son reservorios primarios para los serovares Bratislava, al igual que los equinos y accidentales para Autumnalis (DAMMERT, 2005).

Las leptospiras pueden entrar al cuerpo a través de laceraciones en la piel, el contacto con la mucosa o conjuntiva, la inhalación de aerosoles o la ingestión de alimentos o bebidas contaminadas (MONAHAN et al., 2009; MUSSO y La SCOLA, 2013). La leptospirosis, se considera una enfermedad profesional, que afecta principalmente agricultores, pescadores, veterinarios y trabajadores de alcantarillas y mataderos por su susceptibilidad de adquirirla debido a su alto riesgo de exposición (BROWN et al., 2011). Un riesgo de infección para los seres humanos es la exposición durante actividades recreativas relacionadas con el agua (MONAHAN et al., 2009; MARGA et al., 2013), o riesgo profesional como trabajadores de cultivos de arroz (ROCA, 2006).

En el humano, la sintomatología de la infección es amplia, va desde un simple resfriado hasta el clásico síndrome de la enfermedad de Weil, forma más severa y causada por el serovar Icterohaemorrhagiae (LEVETT, 2001). El inicio de la enfermedad es similar en la forma ictérica y anictérica, pero al cabo de tres a seis días de evolución, los síntomas alcanzan su máxima intensidad (CÉSPEDES, 2005).

En la forma anictérica, hay cefalea intensa, mialgias principalmente de pantorrillas y región lumbar, escalofríos, hiperemia conjuntival, emesis, pirexia $\left(40^{\circ} \mathrm{C}\right)$ en algunos casos dolor ocular, hepatomegalia, esplenomegalia y malestar general con postración, esta presentación es menos severa (CÉSPEDES, 2005). La forma ictérica o síndrome de Weil, es la presentación más severa y más aguda de la enfermedad (MERIEN, 2000) y se caracteriza por falla hepática y renal; la ictericia se debe al aumento de la bilirrubina asociando a coluria y prurito. Hay una elevación de la mayoría de las transaminasas (VIJAYACHARY et al., 2008). Con la instalación de la insuficiencia renal, puede desarrollarse delirio y convulsiones, junto con la aparición de manifestaciones hemorrágicas diversas y acentuación de la ictericia (CÉSPEDES, 2005). La vasculitis afecta preferentemente al hígado y a riñones, pero puede afectar a cualquier órgano. La hipovolemia es debida a la extravasación de los elementos sanguíneos y a la deshidratación concomitante, también contribuyen al deterioro de la función renal (ROCA, 2006). El objetivo general fue conocer la presencia de anticuerpos contra L. interrogans (sensu lato) en trabajadores de granjas porcinas del medio Sinú.

\section{Materiales y métodos.}

Zona de estudio: El trabajo se implementó en 18 granjas porcinas de Montería, Cereté y Ciénaga de Oro; municipios ubicados en la cuenca media del río Sinú, del departamento de Córdoba. La cuenca hidrográfica del río Sinú tiene un área aproximada de 1.395.244 hectáreas de las cuales el 93\% corresponde al departamento de Córdoba, el $6 \%$ a Antioquia y el $1 \%$ al departamento de Sucre. (CVS, 2004).

Tipo de estudio y cálculo del tamaño de la muestra: Se propuso un estudio epidemiológico de tipo transversal que involucró 18 granjas porcinas con manejo tecnificado, ubicadas en tres municipios del medio Sinú.

Toma de sangre en humanos: En cada una de las granjas porcícolas involucradas en el presente estudio, y previo consentimiento por escrito de los propietarios y del personal involucrado, se tomaron 62 muestras de sangre a los trabajadores implicados directamente en el manejo zootécnico, por una bacterióloga, quien desinfectó con alcohol antiséptico al $70 \%$ la región de la vena radial, cubital y/o mediana, donde se extrajeron $5 \mathrm{ml}$. de sangre, las cuales se depositaron en tubos rotulados, manteniéndose en refrigeración hasta el arribo al Instituto de Investigaciones Biológicas del Trópico (IIBT), de la Universidad de Córdoba, donde fueron procesadas de acuerdos a unos protocolos previamente definidos.

Realización de pruebas serológicas (MAT): Las muestras de sangre se centrifugaron a 3000 gravedades por 5 minutos para obtener los sueros, que se conservaron en congelación $\left(-70^{\circ} \mathrm{C}\right)$ hasta la realización del MAT, en el laboratorio del IIBT. 
La detección de anticuerpos frente a $L$. interrogans (sensu lato), se hizo mediante la prueba de microaglutinación (MAT), de acuerdo a las especificaciones de la Organización mundial de la Salud, 2003 (WHO por su sigla en inglés), usando once Serogrupos: Serogrupo Australis (serovar Bratislava), serogrupo Autumnalis (serovar Au0tumnalis), serogrupo Batavie (serovar Batavie), serogrupo Serjoe (serovar Hardjo), serogrupo Grippothyphosa (serovar Grippothyphosa), serogrupo Icterohaemorrhagiae, (serovar Icterohaemorrhagiae), serogrupo Mini (serovar Mini), serogrupo Pomona (serovar Pomona), serogrupo Pyrogenes (serovar Zanoni), serogrupo Tarassovi (serovar Tarassovi) y serogrupo Canicola (serovar Canicola). Títulos $\geq 1: 100$ se consideraron como positivos.

Diseño estadístico: Los datos obtenidos se tabularon en Excel y luego se analizaron por medio de la estadística descriptiva, mediante las medidas de tendencia central, usando el software SAS.

Aspectos éticos: El Comité de Ética del IIBT, adscrito a la Facultad de Medicina Veterinaria y Zootecnia, de la Universidad de Córdoba revisó y aprobó el protocolo del estudio. Se tuvo en cuenta la Resolución 8430/93 del Ministerio de Salud para estudios en humanos. El trabajo se clasificó de mínimo riesgo y se invitó a participar de forma voluntaria a los trabajadores por medio de un consentimiento por escrito.

\section{Resultados y discusión}

Se analizaron 62 sueros humanos asociados a granjas porcinas; el $87.09 \%(n=54)$ correspondieron al sexo masculino y el $12.912 \%(n=8)$ al sexo femenino. La frecuencia de anticuerpos contra $L$. interrogans (sensu lato) en humanos a riesgos (trabajadores) asociados a granjas porcinas en el medio Sinú fue del $75,80 \%(n=47)$ (IC95\% 65,13\% a $86,46 \%$ ), para cualquier serovar con título $\geq 1: 100$; esta seroprevalencia determinada es superior a lo reportado en estudios de riesgo ocupacional en Colombia (Tabla 1).

En los climas tropicales o subtropicales, las condiciones como humedad y las inundaciones (SAAD et al., 2006) crean escenarios favorables para que una zona sea endémica a leptospirosis; estas condiciones ambientales se presentan en el área donde se implementó el estudio (medio Sinú); estos factores ambientales predominantes en la zona, junto con la presencia y relación estrecha de caninos y humanos que se evidenció en este estudio, probablemente facilitan la permanencia y la transmisibilidad de la infección.

Para disminuir la seroprevalencia determinada en este estudio se debe proponer estrategias como el uso protección adecuada en el trabajo, campañas educativas; ya que se estableció que el uso de una dotación botas, overol, guantes, gafas de protección, etc como barrera sanitaria, tiempo de experiencia de los funcionarios de un zoológico, campañas educativas de prevención en trabajadores agrícolas sobre el uso de protección como barrera sanitaria se consideraron como factores de protección contra infecciones por leptospira (ROMERO et al., 2011; McLEAN et al., 2014). A diferencia donde el bajo nivel educativo, la malas condiciones sanitarias en la vivienda, la falta de saneamiento y los bajos ingresos se asociaron con riesgo de infección (JESÚs et al., 2012).

Es importante la implementación continua de campañas educativas con el fin de concientizar y disminuir los riesgos de infección en comunidades; ya que en población a riesgo la incidencia acumulada para las zoonosis en veterinarios de Argentina fue del 34,1\% (MOLINERI et al., 2013), $y$ en un estudio retrospectivo determinaron que hay una correlación directa entre mayor número de casos en animales y casos humanos (MARTÍNEZ et al., 2012).

Tabla 1. Seroprevalencias de $L$. Interrogans reportadas en Colombia, durante los últimos 14 años.

\begin{tabular}{|c|c|c|c|c|}
\hline Departamento & Municipio & Seroprevalencia & Serovares & Referencia \\
\hline Antioquia & Don Matías & $22.4 \%$ & 6 & Ochoa et al. (2000) \\
\hline Atlántico & 23municipios & $9.7 \%$ & 6 & Macías et al. (2005) \\
\hline Córdoba & 8 municipios & $13.1 \%$ & $\lg M$ & Nájera et al. (2005) \\
\hline Antioquia & Apartado & $67.34 \%$ & 14 & Agud/Restrepo (2007) \\
\hline Meta & Villavicencio & $100 \%$ & 8 & Morales et al (2007) \\
\hline Meta & Villavicencio & $18.4 \%$ & 8 & Góngora et al. (2008) \\
\hline Meta & Villavicencio & $19 \%$ & $\operatorname{lgM}$ & Díaz et al. (2008) \\
\hline Córdoba & Libertador & $67.9 \%$ & 6 & Rodríguez (2009) \\
\hline S. Andrés I & S. Andrés & $35 \%$ & 13 & SDS (2009) \\
\hline Meta & Villavicencio & $26.4 \%$ & 8 & Quitián et al. (2009) \\
\hline Boyacá & Tunja & $21.7 \%$ & 6 & Bermúdez et al. (2010) \\
\hline Tolima & * & $6 \%$ & 5 & Romero et al. (2010) \\
\hline
\end{tabular}

*= Lérida, Mariquita, Piedras. 
Esta evidencia serológica ocupacional determinada en el presente estudio, es igualmente reportada en estudios previos como: NÁJERA et al., (2005) en operarios agrícolas, trabajadores de frigoríficos, carniceros, recolectores de basura y alcantarillado en Córdoba (Colombia), quienes detectaron anticuerpos IgM en el $13,1 \%$ de la población evaluada y donde los operarios agrícolas presentaron una mayor prevalencia en comparación con trabajadores dedicados al sacrificio de animales y trabajadores de aseo y alcantarillado $(42,44 \%, 34,01 \%$ y $23,55 \%$ respectivamente).

En humanos de áreas urbanas de Cali, se determinó una seroprevalencia del 23,3\% (IC95\% 18,3 a 28,3) y se encontró una asociación entre seropositividad y el contacto con animales $(p=0,038)$ particularmente cerdos y conejos (FERRO et al., 2006). En Villavicencio, se detectó una alta seroprevalencia mediante IgM en trabajadores piscícolas y de granjas porcinas y se sugiere que el carácter ocupacional de la infección es debido posiblemente por la falta de medidas de higiene y protección laboral (DÍAZ et al., 2008) y en trabajadores del zoológico de Pereira, la seroprevalencia fue del $25 \%$ donde infección con los diferentes serovares de Leptospira se dio por el contacto de los primates neotropicales y los trabajadores (ROMERO et al., 2011).

Se han determinado como factores de riesgo de infección por leptospirosis en Nicaragua, como el vivir en zonas rurales, lavar ropa o bañarse en un río o arroyo y desarrollar algunas actividades ocupacionales, pero no se observó asociación con la exposición a animales domésticos ni a roedores (ASHFORD et al., 2000). En Perú, bañarse o vivir cerca del río, vivir en áreas con pobres condiciones sanitarias y no usar zapatos, (JOHNSON et al., 2004) y en México, una asociación significativa con la presencia de roedores y el contacto con fuentes naturales de agua (VADO et al., 2002).

En Brasil, estudios previos de ALMEIDA et al. (1994) y KO et al. (1999) citados por JESÚS et al. (2012), concluyen que la leptospirosis es una enfermedad profesional asociada a múltiples factores como: pobres condiciones socio-económicas, bajos niveles de educación, malas condiciones de las viviendas, falta de saneamiento y bajos ingresos; condiciones que se asociaron con actividades laborales de limpieza de alcantarillas y actividades agropecuarias (JESÚS et al., 2012). También en el sureste de Brasil, personal militar, tras entrenamiento de campo, donde el entorno antrópico de los bosques tropicales, son el hogar de muchas especies potenciales de reservorios y pueden causar infecciones en humanos (LUPI et al., 2013). A diferencia de los países europeos, donde la mayoría de las infecciones por leptospira en humanos se asociaron con infecciones importadas por actividades deportivas y vacaciones de aventura (GORIS et al.,
2013; LAGI et al., 2013; Van De WERVE et al., 2013). Aunque en Martinica, se ha reportado infecciones por leptospira, después de la práctica de deportes acuáticos (HOCHEDEZ et al., 2013).

La Tabla 2, presenta la seroprevalencia de anticuerpos contra L. interrogans (sensu lato) de acuerdo a lo propuesto por HERRERA y DILAVE (2007).

Tabla 2. Seroprevalencia de anticuerpos contra $L$. interrogans (sensu lato) en trabajadores de granjas porcinas en el medio Sinú.

\begin{tabular}{cccc}
\hline Prevalecía & Titulo & N & Seroprevalencia \% \\
\hline Negativos & No hay o $<1: 100$ & 15 & 24,19 \\
Bajos & $1: 100$ & 6 & 9,68 \\
Moderados & $1: 200$ & 14 & 22,58 \\
Moderados altos & $1: 200$ a 1.400 & 26 & 41,94 \\
Altos & $>1: 800$ & 1 & 1,61 \\
\hline Total & & $\mathbf{6 2}$ & $\mathbf{1 0 0}$ \\
\hline
\end{tabular}

El $24,19 \%$ de los trabajadores no presentaron ningún título para los 11 serogrupos evaluados; el 9,68\% presentaron títulos bajos que pueden corresponder a casos sospechosos o infecciones pasadas; el 22,58\% de los títulos fueron moderados que se pueden relacionar con inicio de infección; el 41,94\% con títulos moderados altos con podrían corresponder a infección probable y títulos altos que se pueden interpretar como infección activa o casos agudos de leptospirosis en el $1,61 \%$.

Se determinó que la mayor serorreactividad en los trabajadores fue el serogrupo Canicola con el $64,52 \%$ (propia de la especie canina). Los caninos han sido considerado como un factor importante para la transmisión de leptospirosis en ambientes urbanos (LOMAR, 2000), y se ha evidenciado su posible papel por la presencia de anticuerpos anti-Leptospira en estos animales (RODRÍGUEZ, 2004). Estudios previos en caninos en esta misma región han reportado este serovar SÁNCHEZ et al. (2010) y ÁLVAREZ et al. (2011); la mayor serroreactividad de este serovar, se puede explicar porque en todas las granjas se evidenció la presencia de caninos en estrecho contacto con los humanos, sugiriendo que esta especie está jugando un papel muy importante en la trasmisión de la leptospirosis. En trabajadores de fincas ganaderas de Yucatán (México), determinaron una mayor frecuencia para el serovar Canicola y sostienen que los caninos tienen una participación muy importante en la infección de este grupo de trabajadores. Igualmente en Querétaro (México), se documentó infección de leptospirosis de un canino con sintomatología a un veterinario y posteriormente la difusión atípica de un veterinario enfermo a un perro (zooantroponosis), escenario 
probable pero que no suele tomarse en cuenta en zonas endémicas (VADO et al., 2014) (Fig. 1).

Figura 1. Seroprevalencia discriminada por serogrupos de $L$. interrogans (sensu lato) en trabajadores de granjas porcícolas del medio Sinú.

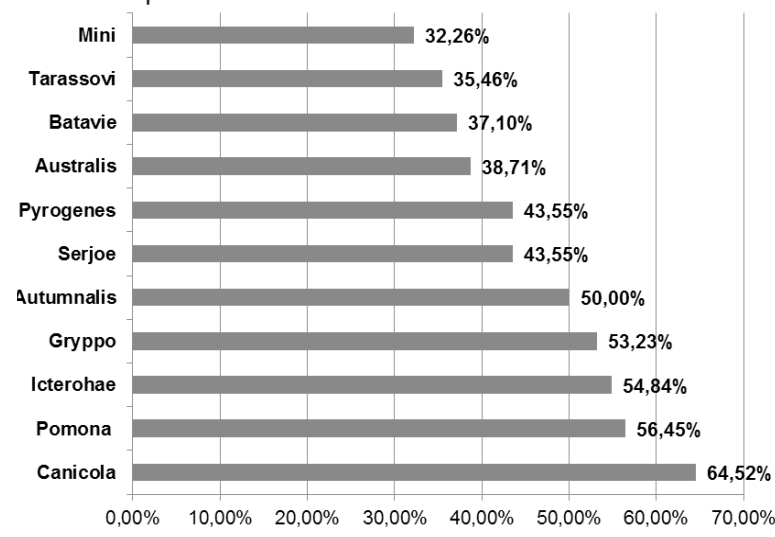

El serogrupo Pomona (propia de la especie porcina), presentó una serorreactividad del $56,45 \%$, lo que estaría indicando que fue la segunda fuente de contaminación de leptospirosis es por el contacto directo con los porcinos. El tercer serovar más frecuente fue Icterohaemorrhagiae con el $54,58 \%$ (propio de los roedores) donde en el $27,78 \%$ (5/18) de las granjas evaluadas se capturaron roedores de las especie Mus musculus, Rattus norvegicus y Rattus rattus, pero fueron seronegativos y no se aisló ninguna cepa (CALDERÓN et al., 2014). En Japón KOIZUMI et al., (2009) aisló cepas de Leptospira de ratas urbanas y afirman que estas continúan siendo importantes reservorios de leptospirosis en zonas urbanas. (SAKUNDARNO et al., 2014). En Indonesia y otros países de Asia y del Pacífico determinaron como factores de riesgo para infección por leptospirosis la presencia de ratas y actividades relacionadas con el contacto con el agua superficial contaminada (SAKUNDARNO et al., 2014)

Títulos del serovar Grippothyphosa, se ha asociado con mapaches, marsupiales y bovinos (FERRO et al., 2006); los serovares Icterohaemorrhagiae y Copenhageni, con ratas, y el serovar Pomona, con ganado porcino y bovino (BARTHI et al., 2003; LEVETT, 2001). Sin embargo, se encontró que en $8,5 \%$ de los sueros tenía anticuerpos frente al serovar Patoc, serovar saprofito. Esta diversidad en la reactividad serológica podría explicarse por el alto grado de reacciones cruzadas que se presentan entre los distintos serogrupos y sus serovares, debido en parte a antígenos comunes entre las leptospiras o por anticuerpos que persisten de contactos infecciosos previos con diferentes leptospiras (TANSUPHASIRI et al., 2005).
Se reporta en esta población humana la presencia de anticuerpos frente a los serovares Mini, Tarassovi, Batavie y Zanoni; el porcentaje de seroprevalencia encontrado fue del $32,78 \%, 36,06 \%, 37,7 \%$ y $39,34 \%$ respectivamente; estos mismos serovares fueron encontrados en caninos y porcinos de estas mismas granjas (datos no publicados). Llama la atención, la respuesta de anticuerpos a los serovares que se encuentran circulando en la zona y la necesidad de realizar una vigilancia epidemiológica que incluya la detección y caracterización de los serovares circulantes.

En Colombia se ha demostrado la circulación de 17 serogrupos lo cual puede deberse a las diferencias ecológicas y ambientales (BELLO et al., 2013). La gran serorreactividad frente a más de un serovar podría explicarse por la presencia de reacciones cruzadas que se presenta entre los diferentes serogrupos y serovares, debido a la presencia de antígenos comunes entre las leptospiras, también puede deberse a la presencia de anticuerpos que persisten de contactos infecciosos previos, anticuerpos IgG anti-leptospira permanecen detectables por meses e incluso años (LEVETT, 2001). Se comprobó aún más la alta endemicidad por ser una región tropical con múltiples reservorios de leptospira y condiciones ambientales favorables para su permanencia. La seroprevalencia en hombres fue del $67,74 \%(42 / 62)$ y en mujeres del $8,06 \%$ (5/47).

La seroprevalencia en hombres fue del $67,74 \%$ (IC95\% 67,74\% a 81,10\%), (42/62) y en mujeres del $8,06 \%$ (IC95\% $115,80 \%$ a $31,92 \%$ ). Se conoce que la prevalencia de leptospirosis es mayor en hombres que en mujeres y se relaciona con actividades géneroespecíficas; en este estudio las labores propias del manejo zootécnico de los porcinos es propia del sexo masculino razón por la cual predominó en la población de estudio y la diferencia encontrada en relación con el sexo femenino no es estadísticamente significativa; sin embargo hay que resaltar que de las ocho mujeres estudiadas 5 presentaron títulos de anticuerpos frente a leptospira. En regiones donde hombres y mujeres trabajan en idénticas condiciones, la tendencia de la enfermedad es a igualarse en los dos sexos; hecho que comprueba la existencia de un factor ocupacional para la enfermedad (FAINE et al., 1999).

Se halló que la mayor seroprevalencia fue del $56,35 \%$ (35/54) se presentó en la categoría de los operarios, que son las personas que están en contacto permanente con los porcinos, seguido de médicos veterinarios zootecnistas y afines con el 4,87\% (3/54) y técnicos agrícolas con el 3,23\% (2/54) esto comprueba aún más la existencia de un factor ocupacional para esta enfermedad (FAINE et al., 1999). 
Cuando se discriminó la presencia o ausencia de sintomatología compatible con leptospirosis, de los 47 individuos seropositivos el 33 (53\%) no presentaron o no recordaron haber presentado sintomatología compatible en los últimos 12 meses; y dentro de los que presentaron síntomas, los más frecuentes fueron: cefalea, mialgias, fiebre los cuales están agrupados en malestar general; igual sintomatología fue reportada por JESÚs et al. (2012).

La ausencia de sintomatología compatible con un cuadro clásico de leptospirosis (ictericia, falla renal, hepática) confirma la alta endemicidad en la zona asociada a infecciones asintomáticas o formas anictéricas caracterizadas por signos y síntomas generales e inespecíficos. Datos similares en el Urabá antioqueño fueron reportados por AGUDELO et al. (2007), quienes encontraron títulos iguales o mayores de 1:400 y plantean al Urabá como de alta endemicidad para leptospirosis y la posibilidad de que la enfermedad se esté presentando en la zona de forma asintomática, subregistrada y asociada con otras enfermedades prevalentes en la zona como dengue y malaria. VIJAYACHARY et al. (2008), afirmaron que la leptospirosis, es una enfermedad subdiagnósticada y que la mayoría de las infecciones son subclínicas. CÉSPEDES (2005) determino que entre el 16-40\% de personas expuestas a fuentes de infección presentan títulos serológicos y no recordaron manifestaciones clínicas sugestivas de esta enfermedad.

Se hacer necesario adelantar más estudios de leptospirosis que contemplen el cultivo y el seguimiento de pacientes ya que VALVERDE et al. (2013) en Puntarenas (Costa Rica) en pacientes hospitalizados con leptospirosis aislaron dos cepas $(\mathrm{JICH} 05$ y INCIENSA 04) estos hallazgos sugieren que varios serotipos virulentos conocidos y desconocidos de Leptospiras están circulando lo que plantea una amenaza para la salud pública.

\section{Conclusión}

Las condiciones ambientales como alta humedad e inundaciones en el Medio Sinú, junto la presencia y relación estrecha de caninos y humanos probablemente facilitan la permanencia y la transmisibilidad de la infección. La frecuencia de anticuerpos contra $L$. interrogans (sensu lato) fue alta que junto con el riesgo ocupacional hace necesario implementar medidas de bioseguridad.

Agradecimientos: Los autores agradecen a la División de Investigaciones y Extensión de la Universidad de Córdoba por la financiación del proyecto.

\section{Referencias}

AGUDELO, P.; RESTREPO, M. 2007. Frecuencia de anticuerpos para 14 serovariedades de Leptospira spp detectados por la prueba de microaglutinación en una serie de casos humanos de Antioquia, Colombia. Rev CES Med 21(2):7-13

ÁlVAREZ, L.; CALDERÓN, A.; RODRÍGUEZ, V.; ARRIETA, G. 2011. Seroprevalencia de leptospirosis canina en una comunidad rural del municipio de Ciénaga de Oro, Córdoba (Colombia). Revista U.D.C.A Actual \& Divulg Cient 14(2):75-81.

ASHFORD, D.A.; KAISER, R.M.; SPIEGEL, R.A.; PERKINS, B.A.; WEYANT, R.S.; BRAGG, S.L.; PLIJAYTIS, B.; JARQUIN, C.; DE LOS REYES, J.O.; AMADOR, J.J. 2000. Asymptomatic infection and risk factors for leptospirosis in Nicaragua. Am J Trop Med Hyg 63(5-6):249-254.

BARTHI, A.R.; NALLY, J.E.; RICALDI, J.N.; MATTHIAS, M.A.; DÍAZ, M.M.; LOVETT, M.A.; LEVETT, P.N.; GILMAN, R.H.; WILLING, M.R.; GOTUZZOE, E.; VINETZ, J.M. 2003. Leptospirosis: a zoonotic disease of global importance. Lancet Infect Dis 3:757-771.

BELLO, S.; RODRÍGUEZ, M.; PAREDES, A.; MENDIVELSO, F.; WALTEROS, D.; RODRÍGUEZ, F.; REALPE, M.E. 2013. Comportamiento de la vigilancia epidemiológica de la leptospirosis humana en Colombia, $2007-2011$. Biomédica 33(1):153-160.

BERMÚDEZ, C.S.; PULIDO, M.M.; ANDRADE, B.R. 2010. Seroprevalencia de Leptospira spp en caninos y humanos de tres barrios de Tunja, Colombia. Rev.MVZ Córdoba 15(3):2193.

BROWN, P.D.; MCKENZIE, M.; PINNOCK, M.; MCGROWDER, D. 2011. Environmental risk factors associated with leptospirosis among butchers and their associates in Jamaica, The International Journal of Occupational and Environmental Medicine 2:47-57. 
CALDERÓN, A.; RODRÍGUEZ, V.; MÁTTAR, S.; ARRIETA, G. 2014. Leptospirosis in pig, dogs, rodents, humans, and water in an area of the Colombian tropics Trop Anim Prod Salud. 46(2):427-432.

CÉSPEDES, Z.M. 2005. Leptospirosis: Enfermedad zoonótica reemergente. Rev Peru Med Exp Salud Publica 22(4):290-307.

CONGRESO DE COLOMBIA. 1989. Ley 84 de 1989. Estatuto Nacional de Protección Animal. Disponible desde Internet en: http:// spac-05. Tripod.com/id24.html Consultado (03/03/2011).

CORPORACIÓN AMBIENTAL DE LOS VALLES DE RIÓ SINÚ Y SAN JORGE. (CVS). 2004. Versión 01. Diagnóstico ambiental de la cuenca hidrográfica del río Sinú. Delimitación, extensión, localización y situación ambiental. Universidad de Córdoba.

DAMMERT, N. 2005. Leptospirosis: Una revisión bibliográfica. Disponible en: http://www.sapuvetnet.org/antigo/Pdf\%20Files/Monografia_leptospira.pdf Consultado (12/08/2010).

DÍAZ, P.L.; ZAPATA, I.; GÓNGORA, O.A.; PARRA, A.J.; APONTE, G.L.; GÓMEZ, LL. 2008. Detección de anticuerpos IgM a leptospira en humanos en riesgo ocupacional en Villavicencio, Meta. Rev.MVZ Córdoba 13(1):1120-1127.

FAINE, S,B.; ALDER, B, BOLIN C, PEROLAT. P. 1999. Appendix 2. Species and serovar list. Leptospira and leptospirosis 2nd ed. Melbourne, Australia, Ed MediSci 193-199.

FERRO, B.E.; RODRÍGUEZ, A.L.; PÉREZ, M.; TRAVI, B.L. 2006. Seroprevalencia de infección por Leptospira en habitantes de barrios periféricos de Cali. Biomédica 26:250-257.

GÓNGORA, A.; PARRA, J.; APONTE, L.; GÓMEZ, L. 2008. Seroprevalencia de Leptospira spp en grupos de población de Villavicencio, Colombia. Rev Salud Pública 10(2):269-278.

GORIS, M.G.A.; BOER, K.R.; DUARTE, T.A.T.E.; KLIFFEN, S.J.; HARTSKEERL, R.A. 2013. Human leptospirosis trends, the Netherlands, 1925-2008. Emerg Infect Dis 19(3):371-378.

GUERRA, M.A. 2013. Leptospirosis: Public health perspectives, Biological Products, 4:295-297.

HERRERA, B.; DILAVE, R.D.M. 2007. Leptospirosis, interpretación de resultados serológicos en animales. Disponible en: http://www.produccion-animal.com.ar/sanidad intoxicaciones metabolicos/enfermedades reproduccion/55interpretacion leptospira.pdf Consultado (12/08/2010).

Consultado (12/08/2010).

HOCHEDEZ P; ESCHER, M.; DECOUSSY, H.; PASGRIMAUD, I.; MARTINEZ, R.; ROSINE, J.; THÉODOSE, R.; BOURHY, p.; PICARDEAU, M.; OLIVE, C.; LEDRANS, M.; CABIÉ, A. 2013. Outbreak of leptospirosis among canyoning participants, Martinique, 2011. Euro Surveill. 18(18) Disponible en:

http://www.eurosurveillance.org/images/dynamic/EE/V18N18/art20472.pdf

Consultado (12/08/2010).

JESÚS, M.S.; SILVA, L.A.; LIMA, K.M.; FERNANDES, O.C. 2012. Cases distribution of leptospirosis in City of Manaus, State of Amazonas, Brazil, 2000-2010. Rev Soc Bras Med Trop 45(6):713-716.

JOHNSON, M.A.; SMITH, H.; JOSEPH, P.; GILMAN, R.H.; BAUTISTA, C.T.; CAMPOS, K. J.; CESPEDES, M, KLASKY, P.; VIDAL,C.; TERRY, H.; CALDERÓN, M.M.; CORAL, C.; CABRERA, L.; PARMAR, P.S.; VINETZ, J.M. 2004. Environmental exposure and leptospirosis, Peru. Emerg Infect Dis 10(6): 1016-1022.

KOIZUMI, N.I.; MUTO, M.; TANIKAWA, T.; MIZUTANI, H.; SOHMURA, Y.; HAYASHI, E.; AKAO, N.; HOSHINO, M.; KAWABATA, H.; WATANABE, H. 2009. Human leptospirosis cases and the prevalence of rats harbouring Leptospira interrogans in urban areas of Tokyo, Japan. JMM 58:1227-1230. 
LAGI, F.; CORTI, T.; MELI, M.; PINTO, A.; BARTOLINI, A. 2013. Leptospirosis acquired by tourists in Venice, Italy. J Travel Med. 20(2):128-30.

LEVETT, P. 2001. Leptospirosis. University of the West Indies. Clinical Microbiology Reviews. 14(2):296-326.

LOMAR, A.V.; DIAMENT, D.; TORRES, J.R. 2000. Leptospirosis in Latin America. Infect Dis Clin North Am 14:23-39.

LUPI, O.; CARVALHO, N.M.A.; AVELAR, K.; ROMERO, C.; BRUNIERA, R.; BRASIL, P. 2013. Cluster of leptospirosis cases among military personnel in Rio de Janeiro, Brazil. Int $\mathrm{J}$ Infect Dis 17:129-131.

MACÍAS, H.J.C.; VERGARA, C.; ROMERO, V.C.; FALCONAR, A.K.I. 2005. Comportamiento de la leptospirosis en el departamento del Atlántico (Colombia), Enero de 1999 a marzo del 2004. Salud Uninorte 20:18-29.

MARGA, G.A.G.; KIMBERLY, R. B.; TAMARA, A.T.E.D.; SUZANNE, J. K.; RUDY, A. H. 2013. Human Leptospirosis Trends, the Netherlands 1925-2008, Emerg Infect Dis 19(3):371-378.

MERIEN, F.; TRUCCOLO, J.; BARANTON, G.; PEROLAT, P. 2000. Identification of a 36-kDa fibronectin-binding protein expressed by a virulent variant of Leptospira interrogans serovar icterohaemorrhagiae. FEMS Microbiol. Lett. 185(1):17-22.

MONAHAN, A.M.; MILLER, I.S.; NALLY, J.E. 2009. Leptospirosis: Risks during recreational activities, J Appl Microbiol 107:707-716.

MORALES, C.R.J.; BRAVO, T.D.; MORENO, V.D.; GÓNGORA, A.; OCAMPO A. 2007. Asociación serológica de la infección por leptospira en humanos, porcinos y roedores en una granja de Villavicencio, Colombia. Orinoquia 11(2):73-80.

MUSSO, D.; LA SCOLA, B. 2013. Laboratory diagnosis of leptospirosis: A challenge. J Microbiol Immunol Infect 46:245-252.

PEDRAZA, M.A.; SALAMANCA, E.E.; ROMÁN, Y.R.; OSPINA, J.M.; PULIDO, M.O. 2012. Seroprevalencia de anticuerpos anti-Leptospira en trabajadores de plantas de sacrificio animal en Boyacá, Colombia. Infectio 16(1):31-36.

QUITIÁN, H.; PARRA, J.; GÓNGORA, O.A.; PARRA, A.J.L.; GALLEGO, J.F.; APONTE, G.L.N. 2009. Seroprevalencia de infección por Leptospira spp. en auxiliares y veterinarios de consultorios de pequeños animales de Villavicencio (Colombia). Salud, Barranquilla 25(1):47-55.

MARTÍNEZ P.; ORTEGA, D.; SALINAS, K. 2012. Evolución de la leptospirosis según el Sistema de Vigilancia Epidemiológica Nacional, Chile 2003-2009. Rev Chilena Infectol 29(6):648-654.

McLEAN, M.; RUSCOE, Q.; KLINE, T.; REY, C.; NESDALE, A . 2014. A cluster of three cases of leptospirosis in dairy farm workers in New Zealand. NZ Med J. 127 (1388):13-20.

MINISTERIO DE SALUD; REPÚBLICA DE COLOMBIA. 1993. Resolución 8430/del 4 de Octubre de 1993. Por la cual se establecen las normas científicas, técnicas y administrativas para la investigación en salud. Disponible en: http://www.dib.unal.edu.co/promocion/etica res 8430 1993.pdf.

Consultado (28/01/2011).

MOLINERI, A.; SIGNORINI, M.L.; PÉREZ, L.; TARABLA, H.D. 2013. Zoonoses in rural veterinarians in the central region of Argentina. Aust J Rural Salud 21(5):285-290.

NÁJERA, S.; ALVIS, N.; BABILONIA, D.; ÁLVAREZ, L.G.; MÁTTAR, S. 2005. Leptospirosis ocupacional en una región del Caribe colombiano. salud pública de México. 47(3):240-244.

OCHOA, J.E.; SÁNCHEZ, A.; RUIZ, I. 2000. Epidemiología de la leptospirosis en una zona andina de producción pecuaria. Rev Panam Salud Pública. 7(5):325-330.

ORGANIZACIÓN MUNDIAL DE LA SALUD (OMS). 2008. Leptospirosis humana: guía para el diagnóstico, vigilancia y control/Organización Mundial de la Salud. Centro Panamericano de Fiebre Aftosa. Rio de Janeiro. Centro Panamericano de Fiebre Aftosa -VP/OPS/OMS. 
RÍOS, R.; FRANCO, S.; MATTAR, S.; URREA, M.; TIQUE, V. 2008. Seroprevalencia de Leptospira sp., Rickettsia sp. y Ehrlichia sp. en trabajadores rurales del departamento de Sucre, Colombia. Infectio 12(2):318-323.

ROCA, B. 2006. Leptospirosis. Rev Med Univ Navarra 50(2):3-6.

RODRÍGUEZ, A.L.; FERRO, B.E.; VARONA, M.X.; SANTAFÉ, M. 2004. Evidencia de exposición previa a Leptospira en perros callejeros de Cali. Biomédica 24:291-295.

RODRÍGUEZ, B.H.; LOZANO, M.C.; BEDOYA, S.C.; GRONDONA, P.L. 2009. Prevalencia de leptospirosis en humanos en la zona urbana del municipio de Puerto Libertador, Córdoba, Colombia. RIAA 0(1):23-28.

ROMERO, M.H.; SÁNCHEZ J.A.; HAYEK, L.C. 2010. Prevalencia de anticuerpos contra Leptospira en humanos urbanos y poblaciones caninas del Departamento de Tolima. Rev. salud pública 12(2): 268-75.

ROMERO, M.; ASTUDILLO, M.; SÁNCHEZ, J.A.; GONZÁLEZ, L.M.; VARELA, N. 2011. Anticuerpos contra Leptospira sp. en primates neotropicales y trabajadores de un zoológico colombiano. Rev. Salud Pública. 13 (5): $814-823$.

SAAD, C.; MORON, L.; PARRA, E.; HIGUERA, L.; PACHECO, A. 2006. Leptospirosis humana: hallazgos clínicos e histopatológicos en un caso y revisión de la literatura. Revista Colombiana de Enfermería 1(1)51-63.

STATISTICALANALYSIS SYSTEM (SAS). Institute SAS. SAS/STAT User's Guide (Release 9.1), Cary: NC, USA; 2001.

SAKUNDARNO, M.; BERTOLATTI, D.; MAYCOCK, B.; SPICKETT, J.; DHALIWAL, S. 2014. Risk factors for leptospirosis infection in humans and implications for public health intervention in Indonesia and the Asia-Pacific region. Asia Pac J Public Health 2014 Jan; 26 (1):15-32.

SÁNCHEZ, G.A.E.; BALLUT, P.J.C.; CALDERÓN, R.A.; RODRÍGUEZ, R.V.C. 2010. Leptospirosis: Enfermedad Endémica en Caninos de Áreas Rurales de Montería. (Córdoba). Orinoquia 14(2):75-81.

SECRETARIA DISTRITAL DE SALUD (SDS). 2009. Estudios de seroprevalencia de dengue y leptospira en el departamento archipiélago de San Andrés providencia y Santa Catalina. Disponible en:

http://www.staffandhealth.com/web/images/pdf/Informe_Final_Estudio_Seroprevalencia.pdf Consultado $(28 / 01 / 2011)$.

TANSUPHASIRI, U.; DEEPRADIT. S.; PHULSUKSOMBATI, D.;TANGKANAKUL, W. 2005. A test strip IgM DotELISA assay using leptospiral antigen of endemic strains for serodiagnosis of acute leptospirosis. J Med Assoc Thai 88:391-398.

VADO, S. I.; CÁRDENAS, M.M.F.; JIMÉNEZ, D. B.; ALZINA, L.A.; LAVIADA, M. H.; SUÁREZ, S. V.; ZABALA, V.J.E. 2002. Clinical epidemiological study of leptospirosis in humans and reservoirs in Yucatán, México. Rev Inst Med Trop Sao Paulo 44:335-40.

VADO, S.I.A.; CÁRDENAS, M.M.F.; PÉREZ, O.C.E.; JIMÉNEZ, D.B.M.G.; ARIAS, L.J.J.; PENICHE, L.G.F. 2014. Leptospirosis: una enfermedad ocupacional en trabajadores que conviven con bovinos seropositivos en el estado de Yucatán. Ciencia y Humanismo en la Salud 1(1):4-13

VALVERDE, M.de L.; GORIS, M.G.; GONZÁLEZ, V.; ANCHIA, M.E.; DÍAZ, P.; AHMED, A.; HARTSKEERL, R.A. 2013. New serovars of Leptospira isolated from patients in Costa Rica: implications for public health. J Med Microbiol 62 (9):1263-1271.

VELASCO, C.O.; RIVAS, S.B: 2010. LEPTOSPIROSIS transmitida del hombre al perro. Enf Inf Microbiol 30(3):106109.

Van De WERVE, C.; PERIGNON, A.; JAURÉGUIBERRY, S.; BRICAIRE, F.; BOURHY, P.; CAUMES, E. 2013. TravelRelated Leptospirosis: A Series of 15 Imported Cases Charlotte. J Travel Med 20(4):228-231

VIJAYACHARY, P.; SUGUNAN, A.P.; SHRIRAM, A.N. 2008. Leptospirosis: an emerging global public health problem. J. Biosci 33(4):557-569. 